\title{
P I 4-04. HIV-SELECTEST EIA and rapid test: a novel assay for differential diagnosis of HIV infections in the face of vaccine-generated antibodies
}

\author{
S Khurana1 ${ }^{1}$ PJ Norris² ${ }^{2}$ MP Busch ${ }^{2}$, K Mlisana ${ }^{3}$, AK Salim³ ${ }^{3}$ E Hunter ${ }^{4}$ and \\ H Golding*5
}

Address: ${ }^{1}$ CBER, Food and Drug Administration, Bethesda, MD, USA, ${ }^{2}$ Blood Systems Research Institute, San Francisco, CA, USA, ${ }^{3}$ Center for the AIDS Programme of Research in South Africa, Durban, South Africa, ${ }^{4}$ Emory Vaccine Research Center, Atlanta, GA, USA and ${ }^{5} \mathrm{CBER}$, FDA, Bethesda, MD, USA

* Corresponding author

from AIDS Vaccine 2009

Paris, France. 19-22 October 2009

Published: 22 October 2009

Retrovirology 2009, 6(Suppl 3):PI92 doi:I0.II86/I742-4690-6-S3-PI92

This abstract is available from: http://www.retrovirology.com/content/6/S3/PI92

(c) 2009 Khurana et al; licensee BioMed Central Ltd.

\section{Background}

All current human immunodeficiency virus (HIV) vaccine candidates contain multiple viral components and elicit antibodies that react positively in licensed HIV diagnostic tests. Thus, vaccine trial participants could be falsely diagnosed as infected with HIV and it is a major public health concern for volunteers who want to participate in future HIV vaccine trials. Additionally, uninfected, seropositive vaccinees may encounter long-term social and economic harms. Moreover, this also interferes with early detection of true HIV infections during preventive HIV vaccine trials. Based on the increased number of HIV vaccines being tested globally, it is essential to differentiate vaccine from virus-induced antibodies.

\section{Methods}

Using a whole-HIV-genome phage display library, we identified conserved sequences in Env-gp41 and Gag-p6 which are recognized soon after infection, do not contain protective epitopes, and are not part of most current HIV vaccines. We established a new HIV-serodetection assay termed HIV-SELECTEST was developed based on these peptides that distinguishes between vaccine-induced antibodies and seroconversion following HIV infections.

\section{Results}

The HIV-SELECTEST performed at $>99 \%$ specificity and sensitivity. Importantly, using seroconversion panels collected from plasma donors early after HIV infection (CHAVI), and from male and female enrollees in the AIEDRP, CAPRISA and ZEHRP cohorts, the HIV-SELECTEST detected HIV antibodies within 2-4 weeks of RNA detection, and performed similarly to currently FDA-licensed tests. A rapid test version of the SELECTEST assay has been developed. This rapid test performs at a comparable level of specificity and sensitivity to the EIA version of the HIVSELECTEST. Importantly, in testing of plasma samples from multiple HIV vaccine trials, uninfected trial participants scored negative, while all intercurrent infections were detected within 1 to 3 months of HIV infection.

\section{Conclusion}

Thus both versions of the HIV-SELECTEST are simple but robust diagnostic tools for easy implementation in HIV vaccine trials and blood banks worldwide. 